Pacific Journal of Mathematics

BOUNDARY CONTINUITY OF SOME HOLOMORPHIC 


\title{
BOUNDARY CONTINUITY OF SOME HOLOMORPHIC FUNCTIONS
}

\section{GLICKSBERG}

\begin{abstract}
For certain bounded domains $D$ in $C^{n}$ any continuous function on $D \cup \partial_{A(D)}$, which is holomorphic on $D$ automatically extends continuously to $D^{-}$.
\end{abstract}

For a bounded domain $D$ in $C^{n}$ let $A(D)$ be the sup normed algebra of functions continuous on $D^{-}$and holomorphic on $D$, and let $\partial=\partial_{A(D)}$ denote its Šilov boundary, so $\partial \subset \partial D$. Of course this inclusion can be proper, and in his thesis [0] A. Aytuna raised the question of whether every bounded continuous function on $\partial \cup D$ holomorphic on $D$ necessarily extends continuously at all points of $\partial D \backslash \partial$. Aytuna showed this held when $n \geqq 2$ for the half-ball $D=$ $\left\{z:|z|<1, \operatorname{Re} z_{1}>0\right\}$, where $\partial D \backslash \partial=\left\{z:|z|<1, \operatorname{Re} z_{1}=0\right\}$ is a union of analytic discs and normal family arguments apply. In fact there are simple domains for which continuous extension fails, as we shall see below ( $(3)$, while it holds rather trivially for starlike domains; our purpose here is to point out some classes of domains for which it holds, and indeed something stronger obtains, by virtue of some elementary function algebra facts combined with the Oka-Weil approximation theorem.

Recall that $K \subset D^{-}$is a peak set for $A(D)$ if there is an $f$ in $A(D)$ with $f(K)=1$ and $|f|<1$ on $D^{-} \backslash K . \quad P(K)$ will denote the closure in $C(K)$ of the analytic polynomials and $H^{\infty}(D)$ the bounded holomorphic functions on $D$.

THEOREM 1. Suppose $\partial D \backslash \partial$ is differentiable and covered by a union of peak sets $K$ for $A(D)$, for each of which

(1.1) $f$ holomorphic near $K$ implies $f \mid K$ is uniformly approximable by polynomials, and

(1.2) $x \in K \backslash \partial$ implies $\left(0, \varepsilon_{x}\right) \nu_{x}+K \subset D$, where $\nu_{x}$ is the inward unit normal to $\partial D$ at $x$, and $x \rightarrow \varepsilon_{x}$ is a positive continuous function on $\partial D \backslash \partial$.

If $h$ is bounded and holomorphic on $D$, and, for one of our peak sets $K_{0}$, has a continuous extension to $D \cup\left(\partial \cap K_{0}\right)$, then $h$ extends continuously to $D \cup K_{0}$.

In particular if $h$ extends continuously to $D \cup \partial$ it extends to an element of $A(D)$; in fact in this case we need not assume $h$ bounded on $D$. Hypothesis (1.1) holds if each peak set $K$ is polynomially convex by the Oka-Weil approximation theorem (cf. [3], 
which will also be our reference for facts on uniform algebras).

An alternative to (1.1) is the assumption that each $K$ provides the spectrum of the (necessarily closed [3]) algebra $A(D) \mid K$ : for then $K$ is the joint spectrum of the coordinate functions and the functional calculus [3, p. 76] applies to assert any $f$ holomorphic near $K$ has $f|K \in A(D)| K$, which is just the property used. (Note that if $D^{-}$is the spectrum of $A(D)$, each $K$ has this property.) Hypothesis (1.2), as we shall see, is one of many which might be used; the assumption that we can move all of $K$ into $D$ can be relaxed considerably in the presence of additional hypotheses, with some complication in the argument; in fact we need only know that (as more smoothness of $\partial D \backslash \partial$ guarantees) near each $x_{0} \in \partial D \backslash \partial$ not too small chunks of each $K$ can be moved along a normal into $D$, with rather more information on our $K_{0}$.

THEOREM 2. Suppose $\partial D \backslash \partial$ is a $C^{2}$ manifold and is covered by a union of peak sets $K$ for $A(D)$ which are polynomially convex. Suppose that for one of these, $K_{0}$, there are $v_{j} \rightarrow 0$ in $C^{n}$ with $v_{j}+K_{0} \subset D$. Then if $h$ is bounded and holomorphic on $D$ and extends continuously to $D \cup\left(\partial \cap K_{0}\right), h$ extends continuously to $D \cup K_{0}$.

More generally, suppose $K_{0}$ only contains polynomially convex subsets $K_{j}$ for which $K_{j}+v_{j} \subset D$ for $v_{j} \rightarrow 0$ in $C^{n}$, while for some $z_{0} \in K_{0} \mid \partial$

(2.1) each probability measure $\lambda$ on $\partial \cap K_{0}$ representing $z_{0}$ on polynomials is a $w^{*}$ cluster point of a bounded sequence $\left\{\lambda_{j}\right\}$, where $\lambda_{j}$ is a complex measure on $K_{j}$ multiplicative on polynomials.

Then any bounded holomorphic $h$ on $D$ continuous on $D \cup\left(\partial \cap K_{0}\right)$ has a unique cluster value at $z_{0}$. (The first assertion follows from the second by taking $K_{j}=K_{0}, \lambda_{j}=\lambda_{\text {.) }}$

1. One of the main function algebra facts we shall use is that if $K$ is a peak set for $A(D)$ then $A(D) \mid K$ is closed in $C(K)$ [3]; another is that $\partial \cap K$ provides a boundary for this algebra. (Any representing measure for $x \in K$ on $\partial$ must be carried by $K$, hence by $\partial \cap K$, as one sees by applying it to $f^{n}$, where $f \in A(D)$ peaks on $K$, and letting $n \rightarrow \infty$.) Both enter our proof of Theorem 1 which has been greatly simplified by T. W. Gamelin, to whom I would like to express my thanks.

Let $E \subset D^{-}$be closed and contain the Šilov boundary $\partial$. The basic step in our proof of Theorem 1 is the more general.

LEMma 1. Suppose $z_{0} \in \partial D \backslash \partial$ lies in the peak set $K$ for $A(D)$, $K$ satisfies (1.1), and $v_{j}+K \subset D$ for a sequence $v_{j} \rightarrow 0$ in $C^{n}$. 
Then if

$(*)$

$$
\limsup _{z \in D \rightarrow z_{0}}|g(z)| \leqq \limsup _{z \in D \rightarrow E}|g(z)|, g \in H^{\infty}(D),
$$

any $f \in H^{\circ}(D)$ which extends continuously at all points of $E \cap K$ extends continuously at $z_{0}$.

For the proof, define $f_{j} \in C(K)$ by $f_{j}(z)=f\left(z+v_{j}\right)$. Because of (1.1) and our hypothesis that $v_{j}+K \subset D$ we know $f_{j}$ is a uniform limit of polynomials on $K$, hence of elements of $A(D) \mid K$; since this algebra is closed, $f_{j} \in A(D) \mid K$. But $f$ extends continuously at all points of $E \cap K$ so the sequence $\left\{f_{j} \mid E \cap K\right\}$ converges uniformly on $E \cap K$, a set which includes the boundary $\partial \cap K$ for $A(D) \mid K$, so that in fact $\left\{f_{j}\right\}$ converges in $A(D) \mid K$. Its limit is the restriction to $K$ of some $g \in A(D)$, and evidently for $w \in E \cap K, g(w)=\lim _{z \in D \rightarrow w} f(z)$. Thus $f-g$ tends to zero at each point of $E \cap K$, and given $\varepsilon>0$, by compactness we have a neighborhood $U$ of $E \cap K$ in $D^{-}$for which $|f-g|<\varepsilon$ on $U \cap D$.

Now let $h \in A(D)$ peak on $K$, and let $V$ be an open neighborhood in $D^{-}$of $E \backslash U$ at a positive distance from $K$. Since $h^{m} \rightarrow 0$ uniformly on $V$ we have an $m$ for which $\left|(f-g) h^{m}\right|<\varepsilon$ on $V$, and the same is true on $U \cap D$ because $|f-g|<\varepsilon$ and $|h| \leqq 1$ there. Thus

$$
\limsup _{z \in D \rightarrow E}\left|(f-g) h^{m}(z)\right| \leqq \varepsilon
$$

so that $\varepsilon \geqq \lim \sup _{z \in D \rightarrow z_{0}}\left|(f(z)-g(z)) h^{m}(z)\right|=\lim \sup _{z \in D \rightarrow z_{0}}|f(z)-g(z)|$ by $\left.{ }^{*}\right)$. Since $\varepsilon$ is arbitrary $g\left(z_{0}\right)$ evidently provides the unique cluster value for $f$ at $z_{0}$, yielding our conclusion.

A simple condition insuring $(*)$ is provided by

LEMMA 2. Suppose that for each sequence $\left\{z_{j}\right\}$ in $D$ converging to $z_{0}$ there are closed sets $\mathscr{K}_{j}$ and $E_{j}$ for which $z_{j} \in \mathscr{K}_{j}, E_{j} \subset \mathscr{K}_{j} \subset$ $D, \quad \lim \sup E_{j} \subset E$, while $\sup \left|f\left(\mathscr{K}_{j}\right)\right|=\sup \left|f\left(E_{j}\right)\right|$, all $f \in H^{\infty}(D)$. Then (*) holds.

Let $M$ denote the left side of $\left(^{*}\right)$ and choose $z_{j}$ in $D$ so that $\left|f\left(z_{j}\right)\right| \rightarrow M, z_{j} \rightarrow z_{0}$. With $E_{j}$ and $\mathscr{K}_{j}$ as above we have $w_{j} \in E_{j} \subset D$ for which $\left|f\left(w_{j}\right)\right| \rightarrow M$, and by hypothesis the $w_{j}$ accumulate only in $E$, so trivially $(*)$ follows.

Now in order to prove Theorem 1 it only remains to verify that we can apply Lemma 2 to any $z_{0} \in K_{0} \mid \partial$ since in the presence of $\left({ }^{*}\right)$ Lemma 1 applies. So suppose $z_{j} \rightarrow z_{0}, z_{j} \in D$, and let $x_{j} \in \partial D$ be nearest $z_{j}$; taking $j$ large we can assume $x_{j}$ lies in $\partial D \backslash \partial$, and in fact in a compact neighborhood of $z_{0}$ in $\partial D \backslash \partial$ so that by (1.2) and 
the continuity of $x \rightarrow \varepsilon_{x}$ for some fixed $\varepsilon>0$

$$
(0, \varepsilon) \nu_{x_{j}}+K_{j} \subset D
$$

for $j \geqq j_{0}$, where $K_{j}$ is a peak set containing $x_{j}$. Now $z_{j}=x_{j}+$ $t_{j} \nu_{x_{j}}$, where $t_{j} \rightarrow 0$ necessarily (indeed $t_{j}=\operatorname{dist}\left(z_{j}, \partial D\right) \leqq \operatorname{dist}\left(z_{j}, z_{0}\right)$ ), and we only have to take $\mathscr{K}_{j}=K_{j}+t_{j} \nu_{x_{j}} \subset D$ and $E_{j}=K_{j} \cap E+$ $t_{j} \nu_{x_{j}}$ : evidently $\lim \sup E_{j} \subset E$ while $\sup \left|f\left(E_{j}\right)\right| \geqq \sup \left|f\left(\mathscr{K}_{j}\right)\right|$ follows from the fact that $K_{j} \cap E \supset K_{j} \cap \partial$ is a boundary for $P\left(K_{j}\right)$, so that by translation $E_{j}$ is a boundary for $P\left(\mathscr{K}_{j}\right)$, which contains $H^{\infty}(D) \mid \mathscr{K}_{j}$ because of (1.1). Our proof of Theorem 1 is now complete.

Note that our use of the differentiability of $\partial D \backslash \partial$ was needed only to allow us to satisfy the hypotheses of Lemma 2; this can be accomplished by various other hypotheses on $D$. For example

Theorem 3. Suppose $D$ is a bounded domain in $C^{n}$ and $K \subset \partial D$ is a peak set for $A(D)$ which is polynomially convex and for which $K+v_{j} \subset D$ for a sequence $v_{j} \rightarrow 0$ in $C_{n}$. Suppose a dense subset of $D$ lies on positive dimensional subvarieties $V$ of $D$ all having $V^{-} \mid$ $V \subset \partial \cup \Delta$, where $\Delta \subset C^{n}$ is compact and disjoint from $K$.

Then any $h \in H^{\infty}(D)$ which has a continuous extension to $D \cup$ $(\partial \cap K)$ has a continuous extension to $D \cup K$.

Here we deduce $\left(^{*}\right)$ for $E=\partial \cup \Delta$ from the maximum principle for varieties, noting that we can restrict ourselves to a dense set of $z$ in $D$ on the left side of $\left({ }^{*}\right)$. So Lemma 1 implies Theorem 3 directly.

We should also note that our proof of Theorem 1 applies equally well to convex $D$, where the fact that $\partial D \backslash \partial$ is a union of polynomially convex peak sets follows from the fact that for each $z_{0} \in \partial D$ one has a $w \in \boldsymbol{C}^{n}$ for which

$$
z \longrightarrow \operatorname{Re}(z, w)
$$

assumes its maximum over $D^{-}$at $z_{0}$, so a multiple of $z \rightarrow \exp (z, w)$ provides an element of $A(D)$ which peaks on a subset $K$ of $\partial D$ containing $z_{0}$; trivially $K$ is convex and thus polynomially convex (via such functions of course). One has only to replace translation along normals by maps

$$
\sigma_{\varepsilon}(z)=(1-\varepsilon) z+\varepsilon z_{0}
$$

where $z_{0} \in D$ is fixed. (But in fact that $f \in H^{\infty}(D)$ continually extendable to all of $D \cup \partial$ has a continuous extension to $D^{-}$for $D$ starlike is trivial: with $z_{0}$ now the star center $f_{\varepsilon}=f \circ \sigma_{\varepsilon} \in A(D)$, and $f_{\varepsilon} \rightarrow f$ uniformly on $\partial$, as $\varepsilon \rightarrow 0$, so that $f_{\varepsilon} \rightarrow g \in A(D)$; since $f_{\varepsilon} \rightarrow f$ 
pointwise on $D, f=g$ on $D$.) Of course convex domains are special cases of those for which, for our $K_{0}$, we have a sequence of holomorphic maps $\sigma_{j}$ of $D^{-}$into $D$ with $\sigma_{j}(k) \rightarrow k$ for each $k \in K_{0} \cap \partial$, and such maps will serve in place of our translations $z \rightarrow z+v_{j}$ in Lemma 1; in particular. Theorem 3 holds if our assumption that $K+v_{j} \subset D$ for $v_{j} \rightarrow 0$ is replaced by the existence of such a sequence $\sigma_{j}$.

Part of our proof of Theorem 1 yields some information even when we have no continuity at the Śilov boundary.

Corollary 4. Let $D$ be as in Theorem 1, or convex, and let $K$ be one of our peak sets. If $f \in H^{\infty}(D)$ then $\mathrm{cl}(f, K)$, the set of cluster values of $f$ at points of $K$, lies in $\mathscr{C}=\mathscr{C}(\operatorname{cl}(f, \partial \cap K))$, the closed convex hull of the set of cluster values at points of $\partial \cap K$.

This follows precisely because (*) holds: if our inclusion were to fail, so some $w \in \operatorname{cl}(f, K) \backslash \mathscr{C}$, (necessarily a cluster value at some $\left.z_{0} \in K_{0} \mid \partial\right)$, then so would $\left(^{*}\right)$ for $\exp \left(e^{i \theta} f\right)$, where $\theta$ is chosen so that $\operatorname{Re}\left(e^{i \theta} w\right)>\sup \operatorname{Re}\left(e^{i \theta} \mathscr{C}\right)$.

2. The proof of Theorem 2 is more involved than that of Theorem 1 because we cannot make as great use of the closure of the algebra $A(D) \mid K$. The tubular neighborhood theorem [2, p. 9] allows us to deduce it from the more general result below, in which $\Pi(x, \delta)$ denotes the square polycylinder of radius $\delta$ about $x$.

THEOREM 2'. Suppose $\partial D \backslash \partial$ is differentiable, and covered by a union of polynomially convex peak sets $K$ for $A(D)$, and for each $x_{0} \in \partial D \backslash \partial$ there are $\varepsilon, \delta, \eta>0$ with $2 \delta>\varepsilon$ for which $\Pi\left(x_{0}, \varepsilon+\delta\right) \cap \partial=$ $\varnothing$, while $x \in \Pi\left(x_{0}, \varepsilon\right) \cap(\partial D \backslash \partial)$ implies

$$
(o, \eta) \nu_{x}+\left(K_{x} \cap \Pi(x, \delta)\right) \subset D
$$

where $K_{x}$ is one of our peak sets containing $x$ and $\nu_{x}$ is again the inward unit normal. Finally suppose that for one of our peak sets $K_{0}$ we have a sequence of polynomially convex subsets $K_{j}$ and $v_{j} \rightarrow 0$ in $C^{n}$ with $v_{j}+K_{j} \subset D$, and for some $z_{0} \in K_{0} \mid \partial$

(2.1) each probability measure $\lambda$ on $K_{0} \cap \partial$ representing $z_{0}$ on polynomials is a $w^{*}$ cluster point of a bounded sequence $\left\{\lambda_{j}\right\}$, where $\lambda_{j}$ is a complex measure on $K_{j}$ multiplicative on polynomials.

Then if $h \in H^{\infty}(D)$ extends continuously to $D \cup\left(\partial \cap K_{0}\right)$ it has a unique cluster value at $z_{0}$.

To begin our proof of Theorem $2^{\prime}$, let $B$ be the uniformly closed 
algebra of functions continuous $D \cup K_{0}$ and holomorphic on $D$, and let $X$ be the closure in its spectrum of $D \cup K_{0}$ (hence of $D$ itself), with $\rho$ the restriction to $X$ of the map dual to $A(D) \rightarrow B$. Since the elements of $B$ are all continuous on $D \cup K_{0}$, the natural injection of that set into $X$ is continuous, $1-1$, while $\rho$ provides a continuous inverse, so $D \cup K_{0}$ is imbedded homeomorphically in $X$. In fact, $D$ forms an open subset of $X$ while $\rho$ is $1-1$ over $D$, as is easily seen [4, p. 421]; moreover $\rho$ is also $1-1$ over $K$ since $^{1)}$ no $\hat{b} \in \hat{B}$ (and hence no element of $C(X)$ ) can separate $\rho^{-1}(z)$ for $z \in K_{0}$ : for if $x \in \rho^{-1}(z)$ and $\left\{z_{\hat{o}}\right\}$ is a net in the dense subset $D$ of $X$ with $z_{\tilde{o}} \rightarrow x$ in $X$ then $z_{j}=\rho\left(z_{j}\right) \rightarrow \rho(x)=z$ in $D^{-}$, so

$$
\hat{b}(x)=\lim \hat{b}\left(z_{\hat{\delta}}\right)=\lim b\left(z_{\hat{o}}\right)=b(z)=\hat{b}(z)
$$

since $b \in B$ is continuous on $D \cup K$.

Of course $\rho$ maps $X$ into, hence onto, $D^{-}$; moreover local maximum modulus and the fact that $D$ is open in $X$ shows $\partial_{B} \cap D=\varnothing$, so $\partial_{B} \subset \rho^{-1}(\partial D)$ since $X$ forms a boundary for $\hat{B}$. In fact I claim $\partial_{B} \subset \rho^{-1}(\partial)$. If not some element $\hat{b}$ of $\hat{B}$ peaks at $x \in \rho^{-1}(\partial D \backslash \partial)$, and letting $x_{0}=\rho(x) \in \partial D \backslash \partial$ we have by hypothesis $\varepsilon, \delta, \eta>0$, with $\delta>2 \varepsilon$, $\Pi\left(x_{0}, \varepsilon+\delta\right) \cap \hat{o}=\varnothing$, and

$$
(0, \eta) \boldsymbol{\nu}_{x}+K_{x} \cap \Pi(x, \delta) \subset D
$$

for any $x$ in $\Pi\left(x_{0}, \varepsilon\right) \cap(\partial D \backslash \partial)$, where $K_{x}$ is one of our peak sets containing $x$. Replacing $b$ by $b^{k}$ for $k$ large we can suppose $|b|<$ $1 / 4$ on $D \backslash \Pi\left(x_{0}, 1 / 2 n \min (\varepsilon, \eta)\right)$, while $|b(z)|>3 / 4$ for some $z \in \Pi\left(x_{0}\right.$, $1 / 2 n \min (\varepsilon, \eta))$. Now let $x \in \partial D$ be nearest $z$, so $z=x+t \nu_{x}, 0<$ $t<1 / 2 \min (\varepsilon, \eta)$, and, since $x \in \Pi\left(x_{0}, \varepsilon\right)$, (1) applies. In particular this says $b\left(\cdot+t \nu_{x}\right)$ is analytic near the polynomially convex set $K_{x} \cap \Pi(x, \delta)$, and so lies in $P\left(K_{x} \cap \Pi(x, \delta)\right)$ by Oka-Weil. But this algebra coincides with the uniform closure of $P\left(K_{x}\right) \mid\left(K_{x} \cap \Pi(x, \delta)\right)$ clearly, whose Šilov boundary, by Rossi's local maximum modulus theorem [3], lies in the topological boundary $\partial_{0}=\partial\left(K_{x} \cap \Pi(x, \delta)\right)=$ $K_{x} \cap \partial \Pi(x, \delta)$ of $K_{x} \cap \Pi(x, \delta)$ in $K_{x}$ (since $\Pi(x, \delta) \subset \Pi\left(x_{0}, \delta+\varepsilon\right)$ misses $\partial)$. Because $t<\varepsilon$ and $\delta>2 \varepsilon, \partial_{0}+t \nu_{x}$ lies in the closure of $D \backslash \Pi\left(x_{0}\right.$, $1 / 2 n \min (\varepsilon, \eta))$, where $|b|<1 / 4$, and so we obtain a contradiction $3 / 4<|b(z)|=\left|b\left(x+t \nu_{x}\right)\right| \leqq \sup \left|b\left(\partial_{0}+t \nu_{x}\right)\right| \leqq 1 / 4$, establishing the claim that $\partial_{B} \subset \rho^{-1}(\partial)$.

Moreover, if $g \in A(D) \subset B$ peaks on $K_{0}$ then $\hat{g}=g \circ \rho \in \hat{B}$ peaks on $\rho^{-1} K_{0}$, while the closed algebra $\hat{B} \mid \rho^{-1} K_{0}$ has, as a boundary, $\partial_{B} \cap$ $\rho^{-1} K_{0} \subset \rho^{-1} \partial \cap \rho^{-1} K_{0}=\rho^{-1}\left(\partial \cap K_{0}\right)$ which is precisely $\partial \cap K_{0}$ since $\rho$ is $1-1$ over $K_{0}$ as we saw earlier.

Now consider our function $h$ holomorphic on $D$ and continuous

${ }^{1}$ Here $^{\wedge}$ is the Gelfand representation of $B$. 
on $D \cup\left(\partial \cap K_{0}\right)$. We shall show

(i) $h$ has a continuous extension $h_{0}$ to $X \backslash \rho^{-1}\left(K_{0} \backslash \partial\right)$;

(ii) all probability measures $\lambda$ on $\partial_{B}$ representing our fixed $z_{0} \in K_{0} \mid \partial$ are multiplicative on the closed subalgebra $B_{0}$ of $C\left(X \backslash \rho^{-1}\left(K_{0} \backslash \partial\right)\right)$ generated by $B$ and $h_{0}$;

(iii) the subset $\partial_{B}$ of $X \backslash \rho^{-1}\left(K_{0} \mid \partial\right)$ forms a boundary for $B_{0}$.

Once these facts are in hand our conclusion follows by noting that if $X_{0}$ is the closure of $X \backslash \rho^{-1}\left(K_{0} \backslash \partial\right)$ in the spectrum $M_{B_{0}}$ (hence that of $D$ as well) and $\rho_{0}$ is the restriction to $X_{0}$ of the map dual to $A(D) \rightarrow B_{0}$, then all points of $\rho_{0}^{-1}\left(z_{0}\right)$, for our fixed $z_{0} \in K_{0} \mid \partial$, are represented by measures $\lambda$ on the boundary $\partial_{B}$ for $B_{0}$ (iii) which lie in the set of measures on $\partial_{B}$ representing $z_{0}$ on $B$; since (ii) says these are all multiplicative on $B_{0}$ they all represent the same functional: if $\lambda$ and $\lambda^{\prime}$ represented distinct functionals we'd have $b \in B_{0}$ with $\lambda(b)=0, \lambda^{\prime}(b)=1$, whence multiplicativity of $\left(\lambda+\lambda^{\prime}\right) / 2$ yields $1 / 2=1 / 2\left(\lambda+\lambda^{\prime}\right)\left(b^{2}\right)=\left(1 / 2\left(\lambda+\lambda^{\prime}\right)(b)\right)^{2}=1 / 4$. Thus $\rho_{0}^{-1}\left(z_{0}\right)$ is a singleton, and of course this implies $h$ has a unique cluster value at $z_{0}$ since if $z_{j} \rightarrow z_{0}, z_{j} \in D$, any cluster value of $\left\{h\left(z_{j}\right)\right\}$ is $\hat{h}(x)$ for some $x \in X_{0}$ with $\rho_{0}(x)=z_{0}$.

So it remains to prove (i)-(iii).

To see (i), note that

$$
X=\rho^{-1}\left(D^{-}\right)=\rho^{-1}(D) \cup \rho^{-1}\left(\partial D \backslash K_{0}\right) \cup \rho^{-1}\left(\partial \cap K_{0}\right) \cup \rho^{-1}\left(K_{0} \mid \partial\right)
$$

so $X \backslash \rho^{-1}\left(K_{0} \mid \partial\right)=\rho^{-1}(D) \cup \rho^{-1}\left(\partial D \backslash K_{0}\right) \cup \rho^{-1}\left(\partial \cap K_{1}\right)$, and we only have to see $h$, as a function on $D \subset X$, has a unique cluster value at each $x \in \rho^{-1}\left(\partial D \backslash K_{0}\right) \cup \rho^{-1}\left(\partial \cap K_{0}\right)$. For the second set this is our hypothesis on $h$, and for the first, if $g \in A(D)$ again peaks on $K_{0}$ then $b=$ $(1-g) h \in B$, so $\hat{b}(x)(1-\hat{g}(x))^{-1}$ provides our unique cluster value at $x \in \rho^{-1}\left(\partial D \backslash K_{0}\right)$.

For (ii) recall that by hypothesis we have $v_{j} \rightarrow 0$ in $C^{n}$ and polynomially convex sets $K_{j} \subset K_{0}$ with $v_{j}+K_{j} \subset D$, while each probability measure $\lambda$ on $\partial \cap K_{0}$ representing $z_{0}$ on $P(K)$ is a $w^{*}$ cluster point of a sequence $\left\{\lambda_{j}\right\}$, where $\lambda_{j}$ is a complex measure on $K_{j}$ multiplicative on polynomials.

As we know any probability measure $\lambda^{\prime}$ representing $z_{0}$ on $B$ and carried by $\partial_{B}$ is carried by $\partial_{B} \cap \rho^{-1}\left(K_{0}\right) \subset \rho^{-1}(\partial) \cap \rho^{-1}\left(K_{0}\right)=$ $\rho^{-1}\left(\rho \cap K_{0}\right)=\partial \cap K_{0}$, and so $\lambda^{\prime}=\lambda$ as above, as a measure representing $z_{0}$ on $A(D)$, hence on $A(D) \mid K_{0} \supset P\left(K_{0}\right)$. If $\sigma_{j}(z)=z+v_{j}$ then trivially the translated measures $\sigma_{j}^{*} \lambda_{j}$ (defined by $\sigma_{j}^{*} \lambda_{j}(f)=$ $\lambda_{j}\left(\left(f \circ \sigma_{j}\right)\right)$ still have $\lambda$ as a $w^{*}$ cluster point (now in the space of measures on $X$ ) since $b \circ \sigma_{j} \rightarrow b$ uniformly on $K_{0} \cap \partial$ and $\left\{\lambda_{j}\right\}$ is bounded. Since $h \circ \sigma_{j}$ is analytic near $K_{j}$, and so in $P\left(K_{j}\right)$, as is $b \circ \sigma_{j}$ for $b \in B$, 


$$
\begin{aligned}
\sigma_{j}^{*} \lambda_{j}\left(h^{n} b\right) & =\lambda_{j}\left(\left(h \circ \sigma_{j}\right)^{n} b \circ \sigma_{j}\right)=\left(\lambda_{j}\left(h \circ \sigma_{j}\right)\right)^{n} \lambda_{j}\left(b \circ \sigma_{j}\right) \\
& =\sigma_{j}^{*} \lambda_{j}(h)^{n} \sigma_{j}^{*} \lambda_{j}(b)
\end{aligned}
$$

whence $\lambda\left(h^{n} b\right)=\lambda(h)^{n} \lambda(b)$, so $\lambda$ is multiplicative on $B_{0}$, and (ii) holds.

Now only (iii), that $\partial_{B} \subset X \backslash \rho^{-1}\left(K_{0} / \partial\right)$ forms a boundary for $B_{0}$, remains to be seen. If $b \in B_{0}$ peaks at $x \in W=X_{0} \mid\left(\partial_{B} \cup \rho_{0}^{-1}\left(K_{0}\right)\right)$ and $U$ is a compact neighborhood of $x$ lying in $W$ then for $n$ sufficiently large $b_{1}=b^{n}(1-g)$ ( $\equiv 0$ on $K_{0}, g$ again our element of $A(D)$ peaking on $K_{0}$ ) is an element of $B$ which assumes its maximum modulus only within $U$, hence not on $\partial_{B}$, so $\partial_{B}$ can not be a boundary for $B$. Thus the peak points for $B_{0}$ in $X_{0}$ lie in $\partial_{B} \cup \rho_{0}^{-1}\left(K_{0}\right)$, so $\partial_{B_{0}} \subset$ $\partial_{B} \cup \rho_{0}^{-1}\left(K_{0}\right)$. Since the dense open subset $D$ of $X$ lies in $X_{0} \backslash \rho_{0}^{-1}\left(K_{0}\right)$, the peak set $\rho_{0}^{-1}\left(K_{0}\right)$ is nowhere dense in $X_{0}$. But now if $V=\partial_{B_{0}}$ l $\partial_{B} \subset \rho_{0}^{-1}\left(K_{0}\right)$ is nonvoid it must contain a peak point $x_{0}$ for $B_{0}$ (as a relatively open subset of $\partial_{B_{0}}$ must), and since the element $1-\hat{g}$ of $\hat{B}_{0}$ vanishes on $\rho_{0}^{-1}\left(K_{0}\right) \supset V$ it must vanish on a neighborhood of $x_{0}$ in $X_{0}$ by $[5,2.1]$, despite the fact that we have seen $(1-\hat{g})^{-1}(0)=$ $\rho_{0}^{-1}\left(K_{0}\right)$ is nowhere dense in $X_{0}$. We conclude $\partial_{B_{0}} \subset \partial_{B}$ so our proofs of (iii) and Theorem $2^{\prime}$ are complete.

A variant of our argument yields another version of Theorem 2.

Theorem 2". Suppose $\partial D \backslash \partial$ is a $C^{2}$ manifold and is covered by a union of peak sets $K$ for $A(D)$ each of which forms the spectrum of $A(D) \mid K$ (which is automatic if $D^{-}$is the spectrum of $A(D)$ ). Suppose that for one of these, $K_{0}, v_{j}+K_{0} \subset D$ for $v_{j} \rightarrow 0$ in $C^{n}$. Then any bounded holomorphic $h$ on $D$ extending continuously to $D \cup\left(\partial \cap K_{0}\right)$ extends continuously to $D \cup K_{0}$.

This replacement of polynomial convexity for our $K$ 's is possible since here $K \cap \Pi(x, \delta)$ is the joint spectrum of the coordinate functions for the algebra $(A(D) \mid K \cap \Pi(x, \delta))^{-}$, so our argument that $\partial_{B} \subset \rho^{-1}(\partial)$ proceeds as before using the holomorphic calculus in place of Oka-Weil; a similar replacement occurs when we consider the functions $h \circ \sigma_{j}$ of course.

Note that when (1.1) in Theorem 1 is replaced by polynomial convexity of the peak sets $K$ that result is contained in the assertion of Theorem 2' (with all $K_{j} \equiv K_{0}$ ). We should also note a property of such domains: for $D$ as in Theorem 1 (or starlike) any $h \in H^{\infty}(D)$ bounded near $\partial$, say by $M>0$, is bounded on $D$, and by the same constant. (If $h$ were not bounded by $M$ then we have $z_{j}$ in $D$ with $\left|h\left(z_{j}\right)\right| \rightarrow \sup |h(D)|>M$, and we can assume $z_{j} \rightarrow z_{0}$, necessarily in $\partial D \backslash \partial$; if $x_{j} \in \partial D$ is nearest $z_{j}$ and we take $j \geqq j_{0}$ all the $x_{j}$ will lie in a compact neighborhood of $z_{0}$ in $\partial D \backslash \partial$ on which 
$\varepsilon_{x} \geqq \varepsilon>0$, so $z_{j}=x_{j}+t_{j} \nu_{x_{j}}$, with $t_{j}<\varepsilon$ for $j \geqq j_{1}$. But now since $K_{x_{j}}+t_{j} \nu_{x_{j}} \subset D$ while $h\left(\cdot+t_{j} \nu_{x_{j}}\right)$ is in $P\left(K_{j}\right)$ as before,

$$
\left|h\left(z_{j}\right)\right| \leqq \sup \left|h\left(K_{x_{j}}+t_{j} \nu_{x_{j}}\right)\right| \leqq \sup \left|h\left(\partial \cap K_{x_{j}}+t_{j} \nu_{x_{j}}\right)\right|
$$

so $M<\sup |h(D)|=\lim \left|h\left(z_{j}\right)\right| \leqq \lim \sup \left|h\left(\partial \cap K_{x_{j}}+t_{j} \nu_{x_{j}}\right)\right| \leqq M$, our contradiction.) As a consequence if $D$ is as in Theorem 1 (or starlike) and $h \in A(D)$ then $h(\partial \cup D)$ provides the entire range of $h$, $h\left(D^{-}\right)$. To see this we need only show $0 \notin h(\partial \cup D)$ implies $0 \notin h\left(D^{-}\right)$. But the hypothesis implies $1 / h$ is holomorphic on $D$ and bounded on (and so near) $\partial$, so $1 / h$ is bounded on $D^{-}$by our remark, and $0 \notin$ $h\left(D^{-}\right)$. (Even when $D^{-}$is the spectrum of $A(D)$, so $h\left(D^{-}\right)$is the spectrum of $h$, the familiar Banach algebra fact that $\partial h\left(D^{-}\right) \subset h(\partial)$, which thus implies $h(D) \cup \partial h\left(D^{-}\right) \subset h(\partial \cup D)$, does not quite yield this since $\partial h(D)$ may properly contain $\partial h\left(D^{-}\right)$.) More generally, for $h \in H^{\infty}(D)$ the set of cluster values of h at all points of $\partial D, \mathrm{cl}(h, \partial D) \subset$ $h(D) \cup \operatorname{cl}(h, \partial)$ for $D$ as in Theorem 1 , by the same argument.

An improvement of Theorem 2 can be obtained via the basic lemma of [1], viz: for $a \in A(D)$ and $E \subset C$ a closed set of (inner logaritmic) capacity zero, if $h$ has a single cluster value at each point of $\partial \cap\left(K_{0} \mid a^{-1}(E)\right)$ the same is true at $z_{0}$ if $z_{0} \in K_{0} \mid a^{-1}(E)$. Here one argues exclusively with Jensen measures. $h_{0}$ is now only continuous on $Y=\left(X \backslash \rho^{-1}\left(K_{0} \backslash \partial\right)\right) \backslash\left(a^{-1}(E) \cap \partial\right)$, but since $\lambda\left(a^{-1}(E)\right)=0$ for each Jensen measure $\lambda$ representing $z \in K_{0} \mid\left(\partial \cup a^{-1}(E)\right.$ ) on $B$ (by [1, Lemma 1]), $h_{0} \circ \sigma_{j} \rightarrow h_{0}$ a.e. $\lambda$, so as before one concludes all such Jensen measures for $z$ coincide on the subalgebra $B_{0}$ of $C(Y)$ generated by $B$ and $h_{0}$, and represent the same functional $\dot{\phi}_{0}$ on $B_{0}$. On the other hand by the proof of (iii) $\partial_{B_{0}}$ is the closure in $M_{B_{0}}$ of $\partial_{B}$ l $a^{-1}(E)$, and it is easy to see the new points lie in $\rho_{0}^{-1} a^{-1}(E)=\hat{\alpha}^{-1}(E)$; since each element of $\rho_{0}^{-1}(z)$ is represented by a Jensen measure $\lambda$ on $\partial_{B_{0}}$, which necessarily vanishes on $\hat{a}^{-1}(E), \lambda$ is in fact carried by $\partial_{B}$, and represents $z$ on $B$. But now $\lambda$ represents $\phi_{0}$, and $\rho_{0}^{-1}(z)$ is a singleton as desired.

3. There are simple domains for which continuous extension fails. Here is one which amounts to a union of two convex domains, deformed so that two discs in the boundary meet in precisely their common center, and thus obstruct continuous extension; as will be noted there is a vast gap between the example and the domains previously considered.

In $C^{3}$ let

$$
\begin{aligned}
& D_{1}=\left\{t\left(z_{1}, 0,2 i\right)+(1-t) w: 0<t<1,\left|z_{1}\right|<1,|w-(0,0,2)|<1\right\}, \\
& D_{2}=\left\{t\left(0, z_{2},-2 i\right)+(1-t) w: 0<t<1,\left|z_{2}\right|<1,|w-(0,0,2)|<1\right\},
\end{aligned}
$$


and $D_{0}=D_{1} \cup D_{2}$. Note that $\pi_{3} D_{0}$ lies in the open right half plane, while $\pi_{3} D_{0}^{-}$meets the imaginary axis in $\pm 2 i$; evidently $\pi_{3}^{-1}(i R) \cap D_{0}^{-}$ is the union of the two closed discs

$$
\Delta_{1}=\left\{\left(z_{1}, 0,2 i\right):\left|z_{1}\right| \leqq 1\right\}, \quad \Delta_{2}=\left\{\left(0, z_{2},-2 i\right):\left|z_{2}\right| \leqq 1\right\} .
$$

Thus if we set $\rho\left(z_{1}, z_{2}, z_{3}\right)=\left(z_{1}, z_{2}, z_{3}^{2}\right)$ and $D=\rho D_{0}$ then $\rho$ maps $D_{0}^{-}$ onto $D^{-}$, and $D_{0}$ biholomorphically onto $D$. In fact $\rho$ is $1-1$ on $D^{-}$except at the centers $(0,0, \pm 2 i)$ of $\Delta_{1}, \Delta_{2}$ both of which map to $(0,0,-4)$, which is precisely $\rho \Delta_{1} \cap \rho \Delta_{2}$. Thus the function $h=\pi_{3} \circ \rho^{-1}$ on $D^{-} \backslash\{(0,0,-4)\}$, which provides the square root of the third coordinate, is continuous, and holomorphic on $D$. Since it yields values near both $\pm 2 i$ in each neighborhood of $(0,0,-4)$ in $D^{-}$, it has no continuous extension to $D^{-}$. Finally $(0,0,-4) \notin \partial_{A(D)}$, (essentially since each $\rho \Delta_{i}$ is an analytic disc), and we are done.

(One can easily modify $D_{0}$ so that $\partial D \backslash \rho \Delta_{1} \cup \rho \Delta_{2}$ lies in $\partial_{A(D)}$; moreover $\rho \Delta_{1} \cup \rho \Delta_{2}$ can be made a peak set (as in the example, where $\left(4-z_{3}\right) / 8$ is the peaking function), and polynomially convex.)

Added in proof (April 1, 1979). I am indebted to H. Alexander for the following simpler (and basically different) example. In $C^{2}$ let $D=\{(z, w):|z|<|w|<1\}$. Then $\partial=\{(z, w):|z|=|w|=1\}$, essentially since any point with $|z|=|w|<1$ lies in a disc where $f \in A(D)$ must be analytic (as $\left.\lim _{r / 1} f(r \cdot, \cdot)\right)$; but $f=z / w$ is a bounded continuous function on $D^{-} \backslash\{(0,0)\}$ analytic on $D$ which has no continuous extension to $D^{-}$since $(0,0)$ lies on too many analytic discs. (For the same reason $(0,0)$ lies in no proper peak set.)

\section{REFERENCES}

0. Aydin Aytuna, Some results on $H^{p}$-spaces on strictly pseudoconvex domains, Thesis, University of Washington, 1976.

1. B. Cole and I. Glicksberg, Jensen measures and a theorem of Rado, J. Functional Anal., to appear.

2. G. B. Folland, Introduction to Partial Differential Equations, Princeton University Press, Princeton, N. J., 1976.

3. T. W. Gamelin, Uniform Algebras, Prentice Hall, Englewood Cliffs, N. J., 1969.

4. I. Glicksberg, Removable discontinuities of A-holomorphic functions, Pacific J. Math., 61 (1975), 417-426.

5. —- Maximal algebras and a theorem of Radó, Pacific J. Math., 14 (1964), 919-941; correction, 19 (1966), 587.

Received January 26, 1977 and in revised form July 14, 1978. Work supported in part by the NSF.

UNIVERSITY OF WASHINGTON

Seattle, WA 98105 


\section{PACIFIC JOURNAL OF MATHEMATICS}

\section{EDITORS}

RICHARD ARENS (Managing Editor)

University of California

Los Angeles, CA 90024

Charles W. Curtis

University of Oregon

Eugene, OR 97403

C. C. MOORE

University of California

Berkeley, CA 94720
J. DUGUNDJI

Department of Mathematics

University of Southern California

Los Angeles, CA 90007

R. FINN and J. MILGRAM

Stanford University

Stanford, CA 94305

\section{ASSOCIATE EDITORS}

\section{E. F. BeCKENBACH}

B. H. NeumanN

F. WOLF

K. YoSHIDA

\section{SUPPORTING INSTITUTIONS}

\author{
UNIVERSITY OF SOUTHERN CALIFORNIA \\ STANFORD UNIVERSITY \\ UNIVERSITY OF HAWAII \\ UNIVERSITY OF TOKYO \\ UNIVERSITY OF UTAH \\ WASHINGTON STATE UNIVERSITY \\ UNIVERSITY OF WASHINGTON
}

The Supporting Institutions listed above contribute to the cost of publication of this Journal, but they are not owners or publishers and have no responsibility for its content or policies.

Mathematical papers intended for publication in the Pacific Journal of Mathematics should be in typed form or offset-reproduced, (not dittoed), double spaced with large margins. Please do not use built up fractions in the text of the manuscript. However, you may use them in the displayed equations. Underline Greek letters in red, German in green, and script in blue. The first paragraph or two must be capable of being used separately as a synopsis of the entire paper. Items of the bibliography should not be cited there unless absolutely necessary, in which case they must be identified by author and journal, rather than by item number. Manuscripts, in triplicate, may be sent to any one of the editors. Please classify according to the scheme of Math. Reviews, Index to Vol. 39. All other communications should be addressed to the managing editor, or Elaine Barth, University of California, Los Angeles, California, 90024.

50 reprints to each author are provided free for each article, only if page charges have been substantially paid. Additional copies may be obtained at cost in multiples of 50 .

The Pacific Journal of Mathematics is issued monthly as of January 1966. Regular subscription rate: $\$ 72.00$ a year (6 Vols., 12 issues). Special rate: $\$ 36.00$ a year to individual members of supporting institutions.

Subscriptions, orders for numbers issued in the last three calendar years, and changes of address should be sent to Pacific Journal of Mathematics, P.O. Box 969, Carmel Valley, CA 93924, U.S.A. Older back numbers obtainable from Kraus Periodicals Co., Route 100, Millwood, NY 10546.

PUBLISHED BY PACIFIC JOURNAL OF MATHEMATICS, A NON-PROFIT CORPORATION

Printed at Kokusai Bunken Insatsusha (International Academic Printing Co., Ltd.). 8-8, 3-chome, Takadanobaba, Shinjuku-ku, Tokyo 160, Japan. 


\section{Pacific Journal of Mathematics}

\section{Vol. 80, No. $2 \quad$ October, 1979}

K. Adachi, On the multiplicative Cousin problems for $N^{p}(D) \ldots \ldots \ldots \ldots 297$

Howard Banilower, Isomorphisms and simultaneous extensions in $C(S) \ldots 305$

B. R. Bhonsle and R. A. Prabhu, An inversion formula for a distributional

finite-Hankel-Laplace transformation ................... 313

Douglas S. Bridges, Connectivity properties of metric spaces.......... 325

John Patton Burgess, A selection theorem for group actions ........... 333

Carl Claudius Cowen, Commutants and the operator equations

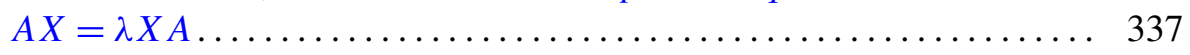

Thomas Curtis Craven, Characterizing reduced Witt rings. II .......... 341

J. Csima, Embedding partial idempotent d-ary quasigroups ............ 351

Sheldon Davis, A cushioning-type weak covering property ............ 359

Micheal Neal Dyer, Nonminimal roots in homotopy trees ............. 371

John Erik Fornaess, Plurisubharmonic defining functions ........... 381

John Fuelberth and James J. Kuzmanovich, On the structure of finitely

generated splitting rings .......................... 389

Irving Leonard Glicksberg, Boundary continuity of some holomorphic

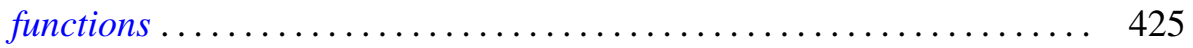

Frank Harary and Robert William Robinson, Generalized Ramsey theory.

IX. Isomorphic factorizations. IV. Isomorphic Ramsey numbers .......

Frank Harary and Allen John Carl Schwenk, The spectral approach to determining the number of walks in a graph...........

David Kent Harrison, Double coset and orbit spaces ..... . .

Shiro Ishikawa, Common fixed points and iteration of commuting

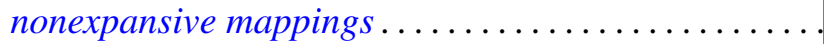

Philip G. Laird, On characterizations of exponential polynomials ........ 503

Y. C. Lee, A Witt's theorem for unimodular lattices ...........

Teck Cheong Lim, On common fixed point sets of commutative

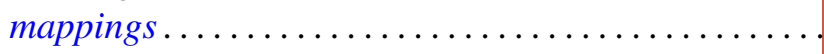

R. S. Pathak, On the Meijer transform of generalized functions ...

T. S. Ravisankar and U. S. Shukla, Structure of $\Gamma$-rings . . .

Olaf von Grudzinski, Examples of solvable and nonsolvable convolution

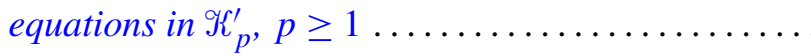

